\title{
Impact of channel constrictions on the formation of multiple tunnel junctions in heavily doped silicon single electron transistors
}

\author{
M. Manoharan, ${ }^{1, a)}$ Shunri Oda, ${ }^{1,2, b)}$ and Hiroshi Mizuta ${ }^{3,4,2}$ \\ ${ }^{1}$ Quantum Nanoelectronics Research Center, Tokyo Institute of Technology, 2-12-1 O-okayama, Meguro-ku, \\ Tokyo 152-8552, Japan \\ ${ }^{2}$ SORST Japan Science and Technology (JST), Japan \\ ${ }^{3}$ School of Electronics and Computer Science, University of Southampton, Highfield, \\ Southampton SO17 1BJ, United Kingdom \\ ${ }^{4}$ Department of Physical Electronics, Tokyo Institute of Technology, 2-12-1 O-okayama, Meguro-ku, \\ Tokyo 152-8552, Japan
}

(Received 11 July 2008; accepted 19 August 2008; published online 16 September 2008)

\begin{abstract}
This paper reports the study of the uncontrolled multiple tunnel junctions (MTJs) induced by the random dopants in heavily doped silicon single electron transistors (SETs). The SETs are fabricated by making dual lateral constrictions in the narrow doped silicon channel formed on a silicon on insulator substrate. The doped SETs with relatively long constriction regions invariably exhibit the MTJ characteristics. The influence of the MTJs is suppressed by tuning the Fermi level in the constriction region. Finally, we show that the formation of uncontrolled MTJs can be avoided by making extremely sharp constrictions. (c) 2008 American Institute of Physics.
\end{abstract}

[DOI: $10.1063 / 1.2980028]$

Silicon based single electron devices (SEDs) have attracted much attention because of their higher operating temperature, and their compatibility with the complementary metal oxide semiconductor technology. ${ }^{1,2}$ Recently, these devices have been more actively studied for qubit ${ }^{3,4}$ and nanoelectromechanical systems. ${ }^{5}$ In particular, a suspended quantum dot cavity structure can be used to study the interaction of single electrons with phonons, the physics of decoherence mechanisms for quantum bits and electromechanical phenomena such as, phononic bandgaps, phonon confinement, quantization of nanomechanical motion, and a strong coupling of nanomechanical and electron motions. ${ }^{5}$ These phenomena may lead to silicon quantum information devices. ${ }^{6}$

One of the common approaches to realizing SEDs in silicon is to use a very narrow doped silicon channel with lateral constrictions in a silicon on insulator (SOI) substrate. ${ }^{7}$ However, these devices often exhibit multiple tunnel junction (MTJ) behaviors irrelevant to their geometrical shapes. ${ }^{8,9}$ These MTJs may smear out the fundamental physical phenomena. The theoretical study in Ref. 10 reports the basic mechanism of the electron island formation and electron transport properties in the heavily doped nanowires, but there has been no experimental work reported to address the MTJ issue in these devices. It is imperative to study the MTJ issue and to develop a fabrication method to avoid these unwanted MTJs to study the fundamental physics of nanodevices.

In this article, we report the measurement results of three heavily doped single electron transistors (SETs) with different channel constriction lengths. It was found that longer channel constriction regions invariably lead to random dopant induced MTJs. The influence of the MTJs decreased with decreasing channel constriction length. Finally, it is demonstrated that it is possible to overcome the uncontrolled MTJ formation by fabricating extremely sharp constrictions with low surface disorder.

\footnotetext{
a) Electronic mail: mano@neo.pe.titech.ac.jp.

${ }^{b)}$ Electronic mail: soda@pe.titech.ac.jp.
}

The device formation process is the same as the SET patterning reported in Ref. 11. A scanning electron microscopy (SEM) image of a typical fabricated SET is shown in Fig. 1(a). The bright regions indicate the SOI layer and the dark regions indicate the BOX layer of the substrate. The lithographically defined constrictions in the silicon channel act as tunnel barriers as a result of bandgap enlargement in the narrower regions. ${ }^{11}$ Higher resolution SEM images of the dot region of devices $\mathrm{A}, \mathrm{B}$, and $\mathrm{C}$ are shown in Figs. $1(\mathrm{~b})-1(\mathrm{~d})$, respectively. The approximate constriction lengths of devices $\mathrm{A}, \mathrm{B}$, and $\mathrm{C}$ are 75,65 , and $45 \mathrm{~nm}$, respectively. Two-thirds of the center dot width was taken into account when calculating the constriction region length [schematically shown in Fig. 1(b)].

Figure 2(a) shows the gray-scale plot of the measured differential conductance as a function of $V_{d}$ and $V_{\mathrm{G} 3}$ with $V_{\mathrm{G} 1}=V_{\mathrm{G} 2}=0 \mathrm{~V}$ for device A at $4.5 \mathrm{~K}$. In the case of a single dot device, Coulomb blockade should be periodically lifted.
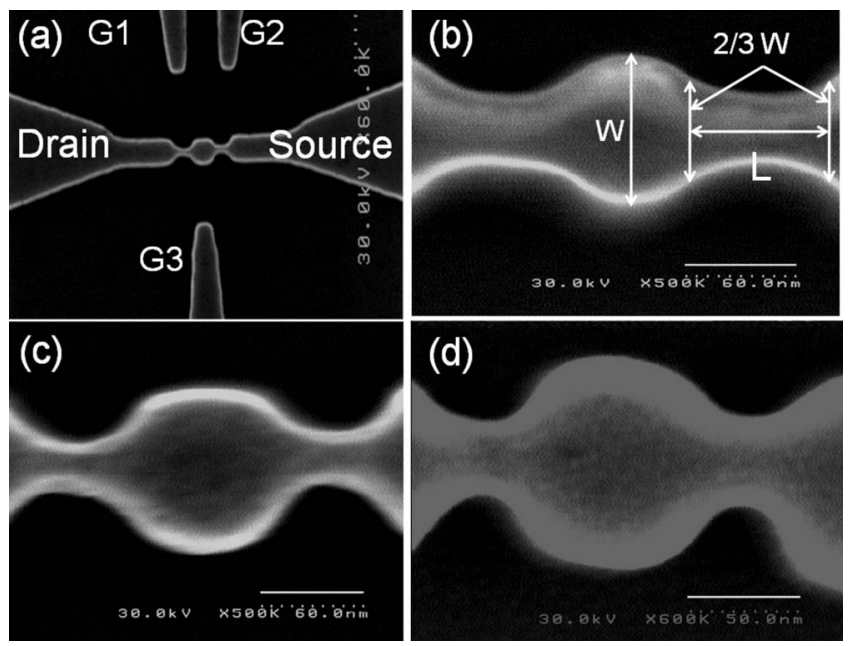

FIG. 1. (a) SEM image of the fabricated SET structure; (b) device A dot region, scale bar: $60 \mathrm{~nm}$; (c) device $\mathrm{B}$ dot region, scale bar: $60 \mathrm{~nm}$; (d) device $\mathrm{C}$ dot region, scale bar: $50 \mathrm{~nm}$. 

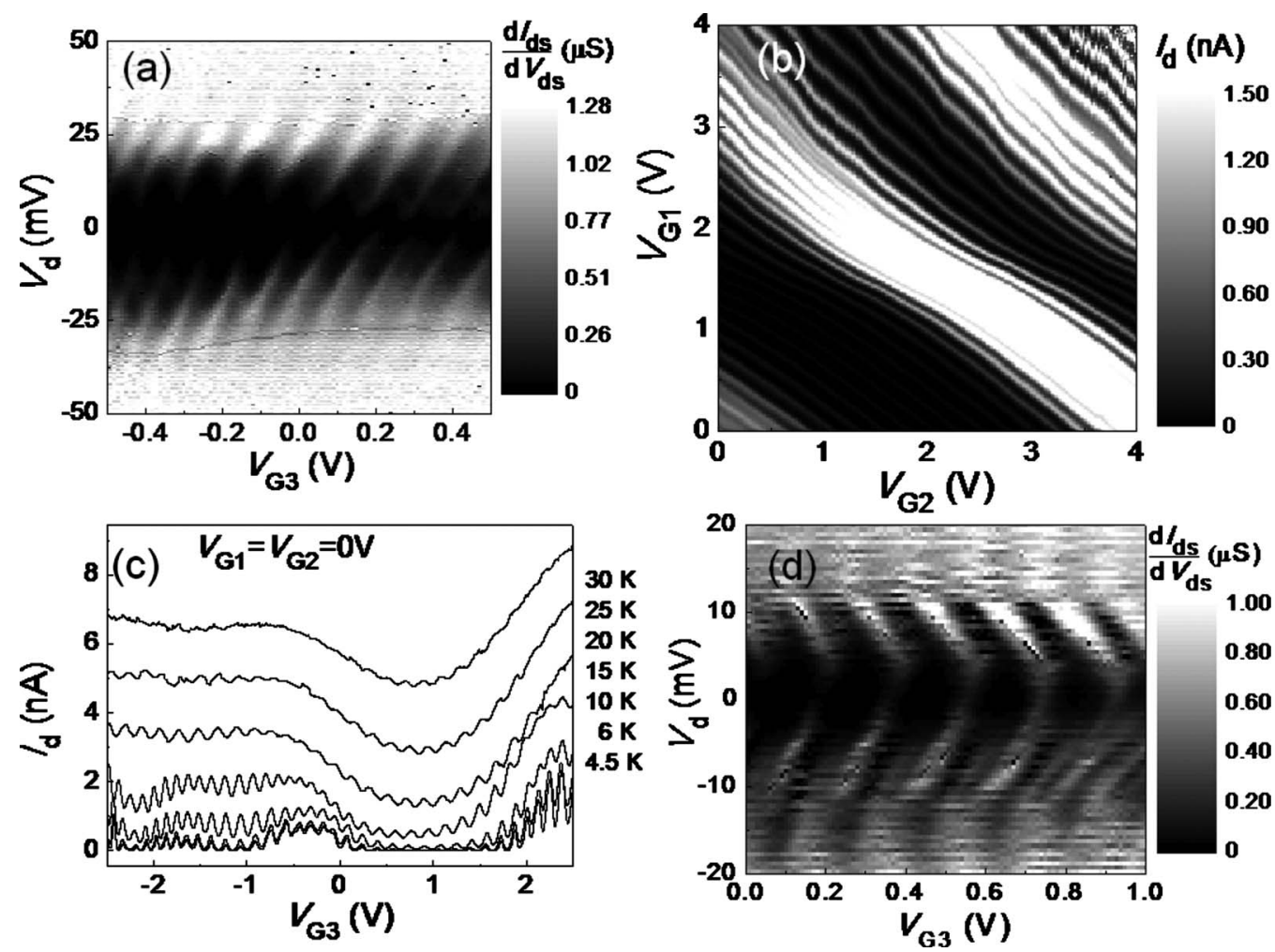

FIG. 2. Device A measurement results: (a) differential conductance gray-scale plot as a function of $V_{d}$ and $V_{\mathrm{G} 3}$ with $V_{\mathrm{G} 1}=V_{\mathrm{G} 2}=0 \mathrm{~V}$ at $4.5 \mathrm{~K}$. (b) Drain current gray-scale plot as a function of $V_{\mathrm{G} 1}$ and $V_{\mathrm{G} 2}$ with $V_{d}=10 \mathrm{mV}, V_{\mathrm{G} 3}=0 \mathrm{~V}$ at $5 \mathrm{~K}$. (c) Drain current plot as function of $V_{\mathrm{G} 3}$ at various temperatures for $V_{d}$ $=10 \mathrm{mV}$, and $V_{\mathrm{G} 1}=V_{\mathrm{G} 2}=0 \mathrm{~V}$. (d) Differential conductance gray-scale plot as a function of $V_{d}$ and $V_{\mathrm{G} 3}$ with $V_{\mathrm{G} 1}=V_{\mathrm{G} 2}=2 \mathrm{~V}$ at $4.2 \mathrm{~K}$.

The measurement results exhibit no periodic blockade lifting, which is attributed to the presence of random dopant induced MTJs in the channel. ${ }^{8}$ The gray-scale plot of the measured drain current of device $\mathrm{A}$ as a function of the gate voltages G1 and G2 is shown in Fig. 2(b) for the drain voltage value of $10 \mathrm{mV}$ at $5 \mathrm{~K}$. Anticrossing characteristics should be apparent in the transport characteristics as a result of the electrostatic coupling between comparable sized dots. ${ }^{12}$ However, no anticrossing characteristics can be noticed from the measurement result, which rules out the presence of multiple dots of comparable size. Also, irregularity in the individual energy level conductance can be observed from this plot. This is due to the MJTs in the channel constriction regions. It should be noted that the irregularity is reduced at higher gate voltages due to the Fermi level tuning, which we discuss later.

Figure 2(c) shows the measured drain current with respect to $V_{\mathrm{G} 3}$ at various temperatures for device A with $V_{\mathrm{G} 1}$ $=V_{\mathrm{G} 2}=0 \mathrm{~V}$ and $V_{d}=10 \mathrm{mV}$. From this plot, it should be noticed that conductance peak positions do not vary with the temperature. This confirms the stable nature of the measured transport characteristics of the lithographically defined dot in the channel. It can be seen that the Coulomb oscillation with the oscillation period of $\sim 125 \mathrm{mV}$ (superimposed on the drain current) gradually disappears with increasing temperature. This uniform Coulomb oscillation is attributed to the geometrically defined dot in the channel. As expected, anomalous oscillation characteristics due to the MTJs induced by random dopants disappear at a higher temperature. Moreover, an examination of the temperature dependence of the Coulomb blockade oscillations shows that the peaks do not split.
By applying certain voltages to constriction region gates, the Fermi level can be increased to a value where only the tunnel barriers due to the geometrical constrictions remain active. ${ }^{9}$ At this condition, as geometrically defined tunnel barriers will only be present in the channel, periodic lifting of the Coulomb blockade has to be noticed. The measured differential conductance characteristics of device A for $V_{\mathrm{G} 1}$ $=V_{\mathrm{G} 2}=2 \mathrm{~V}$ at $4.2 \mathrm{~K}$ is shown in Fig. 2(d), where period lifting of the Coulomb blockade is indeed apparent. This corresponds to the suppression of uncontrolled MTJs by the proper Fermi level tuning in the channel. To further study the impact of constriction in MTJ formation, the constriction length was reduced in other devices. Device B has $\sim 10 \mathrm{~nm}$ shorter channel constriction length than device A. The measured gate G1 Coulomb blockade characteristics with the $V_{\mathrm{G} 2}=2 \mathrm{~V}$ and $V_{\mathrm{G} 3}=0 \mathrm{~V}$ at $5 \mathrm{~K}$ is shown in Fig. 3(a). Although no voltage is applied to gate G3 (compared to device $\mathrm{A}$, where a voltage was applied to tune the Fermi level), periodic Coulomb blockade lifting can be noticed. This indicates that the number of MTJs decreases with a reduction in the channel constriction length.

Figure 3(b) shows the shows the measured drain current of device $\mathrm{C}$ (shortest constriction length device) as a function of the drain and gate $\mathrm{G} 3$ voltages with $V_{\mathrm{G} 1}=V_{\mathrm{G} 2}=0 \mathrm{~V}$ at $4.2 \mathrm{~K}$. A virtually uniform oscillation period indicates that a single charging island is responsible for the Coulomb oscillation (it should be noticed that no voltage was applied to any of the constriction region gates). This is presumably due to the very sharp constrictions, which prevent the formation of the dopant-induced MTJs. Evans et al. ${ }^{10}$ demonstrated that electron islands formed in doped silicon nanowire are quasione-dimensional in nature, with a typical characteristic 
(a)
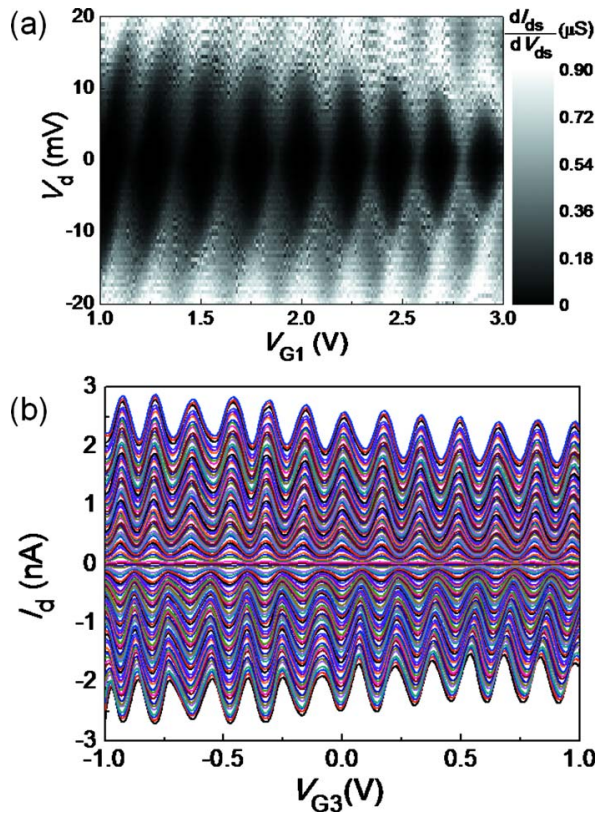

FIG. 3. (Color online) (a) Differential conductance gray-scale plot of device $\mathrm{B}$ as a function of $V_{d}$ and $V_{\mathrm{G} 1}$ with $V_{\mathrm{G} 2}=2 \mathrm{~V}$ at $5 \mathrm{~K}$. (b) Drain current of device $\mathrm{C}$ as a function of $V_{d}$ and $V_{\mathrm{G} 3}$ with $V_{\mathrm{G} 1}=V_{\mathrm{G} 2}=0 \mathrm{~V}$ at $4.2 \mathrm{~K}$. $V_{d}$ varies from $5 \mathrm{mV}$ (top) to $-5 \mathrm{mV}$ (bottom).

length of a few tens of nanometers. The short constriction length in device $\mathrm{C}$ might be below the typical electron island characteristic length, which leads to the avoidance of the formation of uncontrolled dopant induced MTJs.

Figure 4(a) shows the measured drain current at $4.2 \mathrm{~K}$ as a function of $V_{\mathrm{G} 1}$ and drain voltages for device $\mathrm{C}$ with other
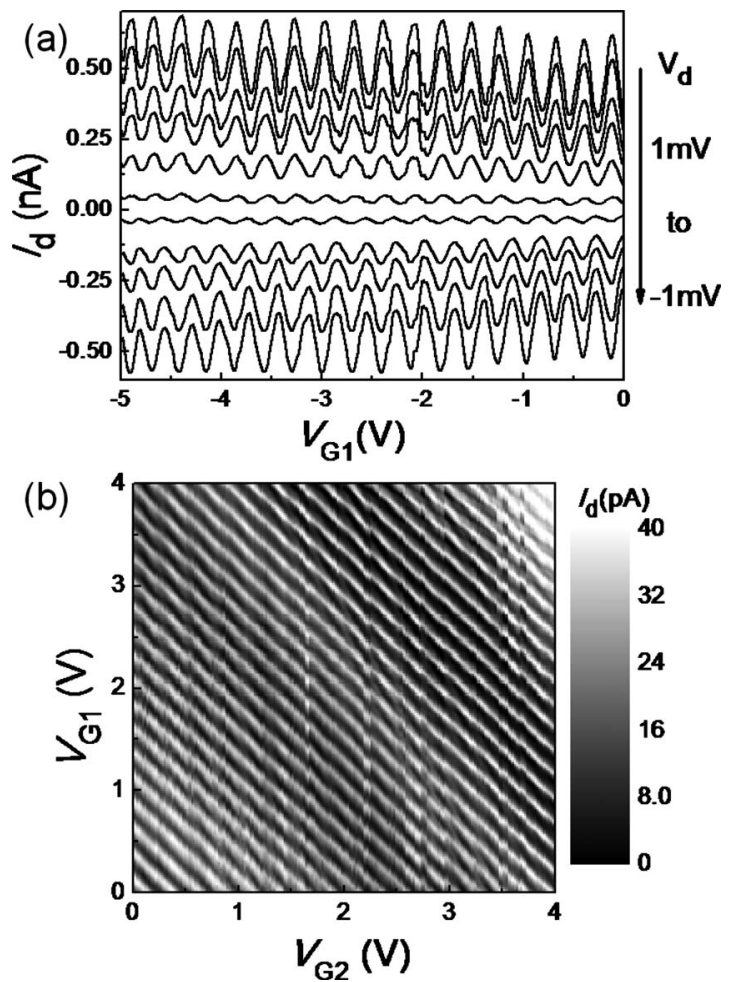

FIG. 4. (a) Drain current as a function of $V_{\mathrm{G} 1}$ for the drain voltages from $1 \mathrm{mV}$ (top) to $-1 \mathrm{mV}$ (bottom) with $V_{\mathrm{G} 2}=V_{\mathrm{G} 3}=0 \mathrm{~V}$ at $4.2 \mathrm{~K}$ for device C. (b) Gray-scale plot of device $\mathrm{C}$ drain current as a function of $V_{\mathrm{G} 1}$ and $V_{\mathrm{G} 2}$ at $4.2 \mathrm{~K}$ with $V_{d}=0.1 \mathrm{mV}, V_{\mathrm{G} 3}=0 \mathrm{~V}$. Vertical lines are due to the artifacts of the measurement system. gates kept at $0 \mathrm{~V}$. A clear Coulomb oscillation with a single oscillation period can be observed over a wide negative gate voltage range. In the case of the negative gate voltage, the Fermi level may be reduced below the barriers of some of the MTJs. However, clear Coulomb oscillation measurement result shows that no MTJs are induced in the negative gate voltage sweep. This clearly indicates that the random dopant induced MTJs can be overcome by making sharp constrictions. The capacitances between G1, G2, G3, and the island were extracted to be $0.59,0.57$, and $1.05 \mathrm{aF}\left(C_{\mathrm{G} 1}, C_{\mathrm{G} 2}\right.$, and $C_{\mathrm{G} 3}$ ), respectively. The fact that $C_{\mathrm{G} 1}$ and $C_{\mathrm{G} 2}$ are almost equal and $C_{\mathrm{G} 3}$ is higher clearly indicates a single dot defined by the geometrical confinement because the gates G1 and G2 are symmetric and distant from the central island while the gate $\mathrm{G} 3$ is closer to it.

To understand the influence of the uncontrolled MTJs on the device transport characteristics, a measurement of the drain current as a function of constriction regions gate voltages $\left(V_{\mathrm{G} 1}\right.$ and $\left.V_{\mathrm{G} 2}\right)$ was carried out. This measurement is shown in Fig. 4(b) for device $\mathrm{C}$ with $V_{d}=0.1 \mathrm{mV}$ and $V_{\mathrm{G} 3}$ $=0 \mathrm{~V}$ at $4.2 \mathrm{~K}$. Parallel current peak lines with about 34 energy levels can clearly be seen. Also, all energy levels are equally coupled (diagonal current peak lines) to the gates G1 and G2. This confirms that a single dot exists between these gates symmetrically, which is the dot defined by the lateral constrictions. If we compare this measurement with device $\mathrm{A}$ result [Fig. 2(b)] then we can understand the influence of MTJs on device transport characteristics. These results clearly indicate that in order to investigate the fundamental properties of silicon quantum dots, MTJs have to be suppressed.

Thus, the doped SETs with relatively long constriction regions invariably exhibit the MTJ characteristics due to the dopant induced potential fluctuation. Decreasing the channel constriction region length along with Fermi level tuning leads to periodic lifting of the Coulomb blockade, which confirms the presence of a geometrically defined single dot. For the device with the sharpest constrictions, the Coulomb blockade is periodically lifted without any Fermi level tuning. This is attributed to the lower constriction length, which might be below the typical dopant induced electron islands characteristic length.

${ }^{1}$ A. Fujiwara and Y. Takahashi, Nature (London) 410, 560 (2001).

${ }^{2}$ Y. Takahashi, M. Nagase, H. Namatsu, K. Kurihara, K. Iwdate, Y. Nakajima, S. Horiguchi, K. Murase, and M. Tabe, Electron. Lett. 31, 136 (1995).

${ }^{3}$ H. W. Liu, T. Fujisawa, Y. Ono, H. Inokawa, A. Fujiwara, K. Takashina, and Y. Hirayama, Phys. Rev. B 77, 073310 (2008).

${ }^{4}$ J. Gorman, D. Hasko, and D. A. Williams, Phys. Rev. Lett. 95, 090502 (2005)

${ }^{5}$ J. Ogi, Y. Tsuchiya, S. Oda, and H. Mizuta, Microelectron. Eng. 85, 1410 (2005)

${ }^{6}$ S. Mahapatra, V. Pod, S. Ecoffey, A. Schmid, C. Washuber, J. W. Tringe, Y. Leblehici, M. Declercq, K. Banerjee, and A. M. Ionescul, Proceedings of the IEDM 2003 (unpublished), p. 703.

${ }^{7}$ Z. Durrani, A. Irvine, H. Ahmed, and K. Nakazato, Appl. Phys. Lett. 74, 1293 (1999).

${ }^{8}$ R. Augke, W. Eberhardt, C. Single, F. E. Prins, D. A. Wharam, and D. P. Kern, Appl. Phys. Lett. 76, 2065 (2000).

${ }^{9}$ C. Single, F. E. Prins, and D. P. Kern, Appl. Phys. Lett. 78, 1421 (2001).

${ }^{10}$ G. J. Evans, H. Mizuta, and H. Ahmed, Jpn. J. Appl. Phys., Part 1 40, 5837 (2001)

${ }^{11}$ Y. Kawata, M. A. H. Khalafalla, K. Usami, Y. Tsuchiya, H. Mizuta, and S Oda, Jpn. J. Appl. Phys., Part 1 46, 4386 (2007).

${ }^{12}$ W. G. van der Wiel, S. De Franceshi, J. M. Elzerman, T. Tarucha, and L. P. Kouwenhoven, Rev. Mod. Phys. 75, 1 (2003). 